\title{
Monitoring of corrosion Potential and Mechanical Resistance of Contaminated Concrete Exposed to a Chlorinated Environment
}

\author{
M.A.B. Zamora, ${ }^{1, *}$ D.N. Mendoza, ${ }^{1}$ H.H. Zamora, ${ }^{2}$ F.A. Calderón ${ }^{3}$ \\ ${ }^{I}$ Facultad de Ingeniería Civil - Xalapa, Universidad Veracruzana, \\ Circ. G. Aguirre Beltrán S/N, Xalapa, Veracruz, México, CP 91090 \\ ${ }^{2}$ Facultad de Ingeniería Industrial. UPA. Altamira, Tamaulipas, México. \\ ${ }^{3}$ CIMAV S.C. Miguel de Cervantes 120 Chihuahua, Chih. México
}

Received 25 April 2008; accepted 23 October 2008

\begin{abstract}
The electrochemical and mechanical behavior were evaluated for specimens of concrete clean and contaminated with 2 and $4 \%$ of $\mathrm{NaCl}$ (of the weight of cement), elaborated with two types of cements: compound and waterproof. The dosage of the mixture of concrete was done on the basis of weight in two 0.45 and 0.65 water/cement ratios. The specimens under study were exposed in two media: clean water and $3 \%$ of $\mathrm{NaCl}$ solution. The test to evaluate the presence of corrosion of the reinforced steel consisted in the monitoring of the half cell potentials as the norm ASTM C-876-91 indicates. For the mechanical behavior the compression test was used, according to the ASTM C-39/C 39-M-99 norms. This paper presents the results obtained for the first 4 months of monitoring, identifying the influence of the attack of chlorides in the corrosion of steel and its mechanical properties. There is no a significant influence of the type of cement on the parameters of the study.
\end{abstract}

Keywords: concrete, steel, corrosion, potentials, chlorides.

\section{Introducción}

La corrosión del acero de refuerzo es la causa más importante del deterioro prematuro de las estructuras de hormigón reforzado [1]. Estudios en E.U.A por parte de la NACE muestran que en 1998, en el territorio estadounidense, tan solo en lo que se refiere a puentes, los cálculos de costos directos anuales ascendían a US \$3.800 millones [2]. El costo del ciclo de vida se ha convertido en un factor muy importante para los ingenieros constructores y profesionales en reparación, así como para las dependencias responsables de las estructuras, muelles, puentes,

\footnotetext{
* Corresponding author. E-mail address: mbaltazar@uv.mx
} 
etc., debido al incremento en gastos de mantenimiento y reparación anticipadas [3].

El acero embebido en hormigón se encuentra protegido contra la corrosión, por pasivación provocada por la alcalinidad del agua en la disolución ( $\mathrm{pH}=12,2$ ó superior), que penetra a través de los poros del hormigón. La alta alcalinidad del medio de un hormigón de buena calidad, hace que se forme una película pasiva sobre la superficie del acero embebido, la cual normalmente previene la disolución del acero [4]. Sin embargo, debido al comportamiento del hormigón como una membrana semipermeable, se produce el ingreso de sustancias o iones agresivos (cloruros, sulfatos) [5-6]. La presencia de estas sustancias agresivas, principalmente los cloruros, junto con el $\mathrm{pH}$ del medio, desempeñan un papel muy importante en el fenómeno de la corrosión [7].

Cuando las condiciones de servicio cambian y el hormigón se altera, o cuando a través de él penetran sustancias agresivas, de las cuales la penetración de los iones cloruro se considera la más importante [8], la corrosión de las varillas se desencadena con una triple consecuencia:

a) el acero disminuye su sección o incluso se convierte completamente en óxido

b) debido a las presiones que ejerce el óxido al generarse, el hormigón puede fisurarse o desintegrarse

c) la adherencia varilla-hormigón disminuye o desaparece.

Para muchos investigadores, el parámetro más importante que activa la corrosión en el acero de refuerzo es la concentración de cloruros libres, totales o por la relación $\left(\mathrm{Cl}^{-} / \mathrm{OH}^{-}\right)$. Pero hay que tener en cuenta que en el inicio de la corrosión influyen diferentes factores como son, entre otros, las proporciones de los hormigones, el tipo de cemento, calidad de los agregados, relación agua/cemento, humedad relativa, condiciones de la superficie y medio ambiente [9].

Se han estudiado acciones correctivas tales como el uso de inhibidores de corrosión, recubrimiento epóxico de las varillas, eliminación electroquímica de los cloruros, que en ocasiones han fallado en proveer una protección satisfactoria para el acero de refuerzo [10]. La utilización de un cemento especial de tipo impermeable también se considera una acción preventiva para estructuras que estén expuestas a medios agresivos, como muelles, puentes, edificios, lo que traería como resultado un periodo de vida útil o de servicio mayor y con ello una reducción en los costos de mantenimiento preventivo y correctivo.

\section{Metodología experimental}

\section{Materiales para la elaboración del hormigón}

- Cemento CPC 30R y Cemento CPC 30R Impermeable [11].

- Agregado Grueso: Grava volcánica de $24 \mathrm{~mm}$.

- Peso específico $(\mathrm{Ss})=2.76$, Absorción = 0.5\% .

- Agregado fino: Arena cuarzosa, Modulo de Finura $(\mathrm{MF})=3.5$.

- Peso específico $(\mathrm{Ss})=2.63$, Absorción = $2.2 \%$.

- Agua: Potable. 


\section{Diseño de mezclas y elaboración de especimenes}

Se diseñaron dos mezclas diferentes de hormigón con relación agua/cemento = 0.45 y 0.65 [12]. La Tabla 1 muestra la dosificación de ambas. Los especimenes (cilindros de hormigón de $15 \times 30 \mathrm{~cm}$ ), se elaboraron de acuerdo con las normas ASTM C470-87 y C192-81.

Tabla 1. Dosificación de las mezclas de hormigón.

\begin{tabular}{|c|c|c|}
\hline Materiales / kg & Rel. a/c 0.45 & Rel. a/c 0.65 \\
\hline Agua & 204 & 204 \\
\hline Cemento & 456 & 316 \\
\hline Grava & 477 & 477 \\
\hline Arena & 1269 & 1415 \\
\hline
\end{tabular}

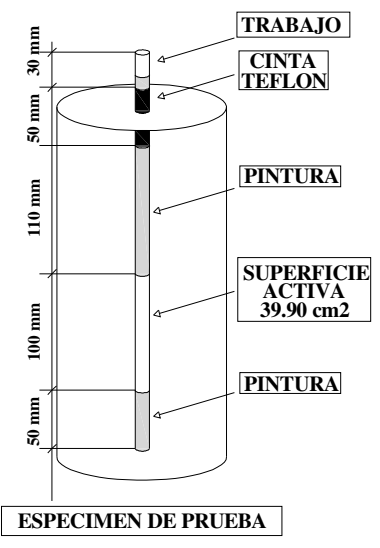

Figura 1. Espécimen de hormigón.

Tabla 2. Nomenclatura especimenes para evaluar la corrosión

\begin{tabular}{|c|c|c|c|}
\hline \multicolumn{2}{|c|}{ Relación a/c $=\mathbf{0 . 4 5}$} & \multicolumn{2}{c|}{ Relación a/c =0.65 } \\
\hline $4 \mathrm{IBC}$ & $4 \mathrm{PBC}$ & $6 \mathrm{IBC}$ & $6 \mathrm{PBC}$ \\
\hline $4 \mathrm{IBL}$ & $4 \mathrm{PBL}$ & $6 \mathrm{IBL}$ & $6 \mathrm{PBL}$ \\
\hline $4 \mathrm{I} 2 \mathrm{C}$ & $4 \mathrm{P} 2 \mathrm{C}$ & $6 \mathrm{I} 2 \mathrm{C}$ & $6 \mathrm{P} 2 \mathrm{C}$ \\
\hline $4 \mathrm{I} 2 \mathrm{~L}$ & $4 \mathrm{P} 2 \mathrm{~L}$ & $6 \mathrm{I} 2 \mathrm{C}$ & $6 \mathrm{P} 2 \mathrm{~L}$ \\
\hline $4 \mathrm{I} 4 \mathrm{C}$ & $4 \mathrm{P} 4 \mathrm{C}$ & $6 \mathrm{I} 4 \mathrm{C}$ & $6 \mathrm{P} 4 \mathrm{C}$ \\
\hline $4 \mathrm{I} 4 \mathrm{~L}$ & $4 \mathrm{P} 4 \mathrm{~L}$ & $6 \mathrm{I} 4 \mathrm{~L}$ & $6 \mathrm{P} 4 \mathrm{~L}$ \\
\hline
\end{tabular}

- 4 y 6 indican la relación a/c 0.45 y 0.65 .

- I y $\mathrm{P}$ se refieren al tipo de cemento, impermeable y normal compuesto respectivamente.

- B indica un hormigón limpio, el 2 un hormigón contaminado con $2 \%$ de $\mathrm{NaCl}$ y el 4 un hormigón contaminado con el $4 \%$ de $\mathrm{NaCl}$. 
- C y L representan los medios de exposición: C para un medio contaminado con $3 \%$ de $\mathrm{NaCl}$ y L para un medio limpio.

Tabla 3. Nomenclatura especimenes resistencia a la compresión.

\begin{tabular}{|c|c|c|c|}
\hline \multicolumn{2}{|c|}{ Relación a/c $=\mathbf{0 . 4 5}$} & \multicolumn{2}{c|}{ Relación a/c $=\mathbf{0 . 6 5}$} \\
\hline $4 \mathrm{IB}-7$ & $4 \mathrm{~PB}-7$ & $6 \mathrm{IB}-7$ & $6 \mathrm{~PB}-7$ \\
\hline $4 \mathrm{IB}-28$ & $4 \mathrm{~PB}-28$ & $6 \mathrm{IB}-28$ & $6 \mathrm{~PB}-28$ \\
\hline $4 \mathrm{IB}-90$ & $4 \mathrm{~PB}-90$ & $6 \mathrm{IB}-90$ & $6 \mathrm{~PB}-90$ \\
\hline $4 \mathrm{I} 2-7$ & $4 \mathrm{P} 2-7$ & $6 \mathrm{I} 2-7$ & $6 \mathrm{P} 2-7$ \\
\hline $4 \mathrm{I} 2-28$ & $4 \mathrm{P} 2-28$ & $6 \mathrm{I} 2-28$ & $6 \mathrm{P} 2-28$ \\
\hline $4 \mathrm{I} 2-90$ & $4 \mathrm{P} 2-90$ & $6 \mathrm{I} 2-90$ & $6 \mathrm{P} 2-90$ \\
\hline $4 \mathrm{I} 4-7$ & $4 \mathrm{P} 4-7$ & $6 \mathrm{I} 4-7$ & $6 \mathrm{P} 4-7$ \\
\hline $4 \mathrm{I} 4-28$ & $4 \mathrm{P} 4-28$ & $6 \mathrm{I} 4-28$ & $6 \mathrm{P} 4-28$ \\
\hline $4 \mathrm{I} 4-90$ & $4 \mathrm{P} 4-90$ & $6 \mathrm{I}-90$ & $6 \mathrm{P} 4-90$ \\
\hline
\end{tabular}

- 4 y 6 indican la relación a/c 0.45 y 0.65 .

- I y P se refieren al tipo de cemento impermeable y normal compuesto, respectivamente.

- B indica un hormigón limpio, el 2 un hormigón contaminado con $2 \%$ de $\mathrm{NaCl}$ y el 4 un hormigón contaminado con el $4 \%$ de $\mathrm{NaCl}$.

- 7, 28 y 90 indican los días en que fueron ensayados a la compresión.

\section{Características del acero de refuerzo}

En cada espécimen se embebió una varilla corrugada de acero 1018 de 3/8" de diámetro, como electrodo de trabajo, colocándoles una franja de $5 \mathrm{~cm}$ de cinta de fluorocarbono, en la parte superior, para evitar que se presenten zonas de aireación diferencial, concentración de sales o corrosión por hendidura [13-14]; además, se pintaron con pintura epóxica para tener un área determinada susceptible a la corrosión (ver Fig. 1)

\section{Descripción de la nomenclatura}

Para la identificación de los diferentes parámetros de estudio, se asignó una nomenclatura a los especimenes para la monitorización de corrosión (Tabla 2) y otra a los especimenes para evaluar su comportamiento mecánico (Tabla 3), como se indica a continuación:

\section{Arreglo de la celda electroquímica}

Los especimenes estuvieron expuestos en dos medios: agua limpia de la red y el agresivo, disolución al $3 \%$ de $\mathrm{NaCl}$. Los resultados que se presentan son de los primeros 4 meses de experimentación: un mes de etapa de curado y 3 meses de exposición. Los potenciales de corrosión o de media celda se midieron de acuerdo con la norma ASTM C-876-91[15], utilizando un electrodo de referencia 
cobre-sulfato de cobre, $\mathrm{Cu} / \mathrm{CuSO}_{4}$. El dispositivo experimental se muestra en la Fig. 2.

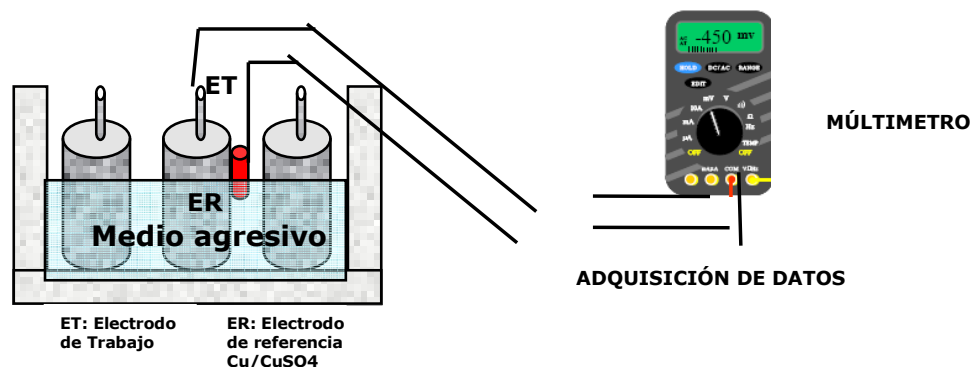

Figura 2. Celda electroquímica de experimentación.

\section{Resultados y discusión}

\section{Resistencia a la compresión}

Se realizaron pruebas de resistencia a la compresión axial sin confinamiento, de acuerdo con la norma ASTM C39-03 [16], a los 7, 28 y 90 días. Los resultados se presentan en las Fig. 3 y 4.

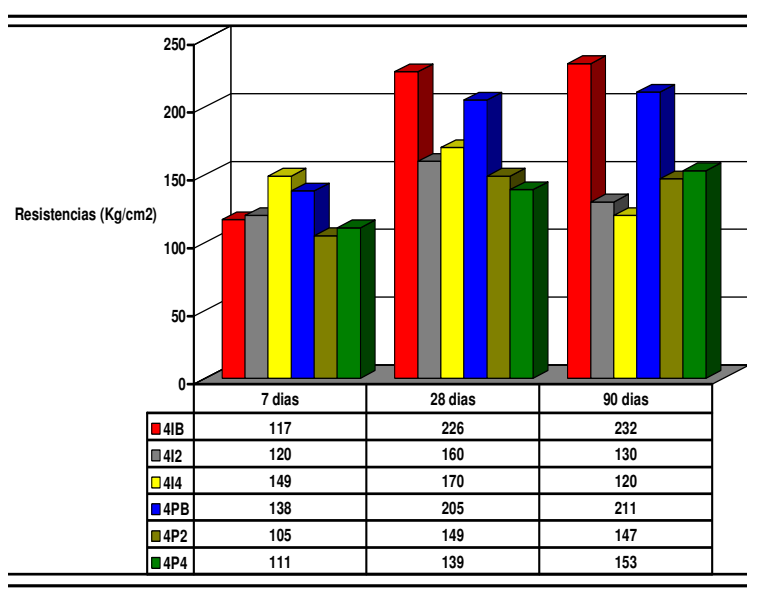

Figura 3. Resistencia a la compresión hormigón relación a/c $=0.45$.

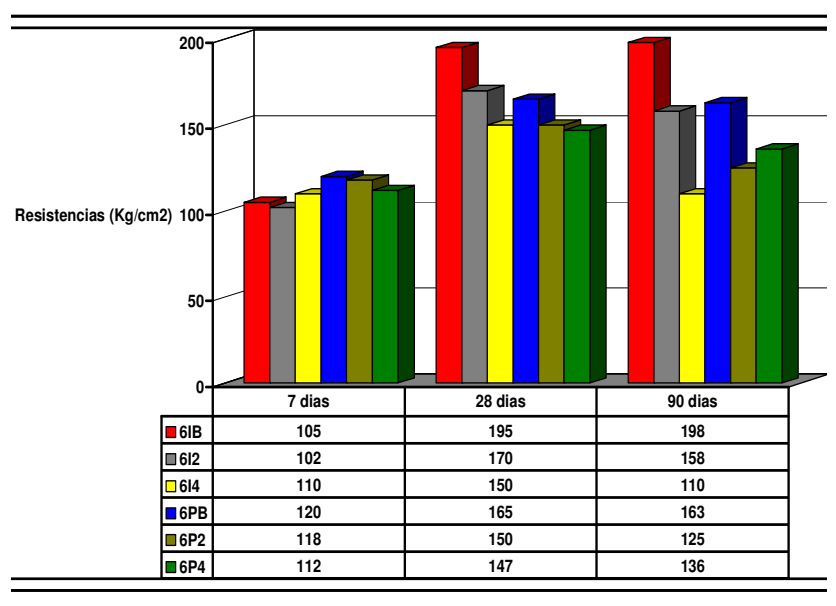

Figura 4. Resistencia a la compresión hormigón relación a/c $=0.65$.

Se observa que los hormigones contaminados con 2 y $4 \%$ de $\mathrm{NaCl}$, sufrieron una disminución en su resistencia a la compresión superior al $20 \%$ en ambas relaciones a/c 0.45 y 0.65 , tanto para el cemento compuesto normal como para el impermeable. Esta disminución es muy significativa dado que es el parámetro que rige, hasta estos momentos, el diseño de las estructuras de hormigón reforzado que se edifican en todo el mundo. 


\section{Potenciales de corrosión}

La monitorización de potencial de media celda en los especimenes se hizo en la zona central como recomienda la literatura [17]. La interpretación de los valores de los potenciales de corrosión se basó en la norma ASTM C876-91 (ver Tabla 4).

Tabla 4. Potenciales de corrosión en hormigón reforzado.

\begin{tabular}{|c|c|}
\hline \multicolumn{2}{|c|}{$\begin{array}{c}\text { Potenciales de corrosión } \\
\text { Norma ASMT C876-91 }\end{array}$} \\
\hline Potenciales más positivos que & $\begin{array}{c}\text { Riesgo de que ocurra } \\
\text { corrosión es del 10\% }\end{array}$ \\
$-200 \mathrm{mV}$ & se tiene una incertidumbre \\
\hline Si está entre $-200 \mathrm{y}-350 \mathrm{mV}$ & $\begin{array}{c}\text { se tendrá una probabilidad de } \\
\text { que se esté corroyendo el } \\
\text { acero, del } 90 \%\end{array}$ \\
\hline Si son más negativos & que -350 mV
\end{tabular}

En la Fig. 5 se observa una tendencia de todos los especimenes a presentar potenciales de corrosión que, de acuerdo a la norma ASTM C-876-91, indican una incertidumbre de que se esté desarrollando la corrosión, teniendo los especimenes con relación 0.45 los valores más positivos, lo cual indica un mejor comportamiento que los de 0.65 .

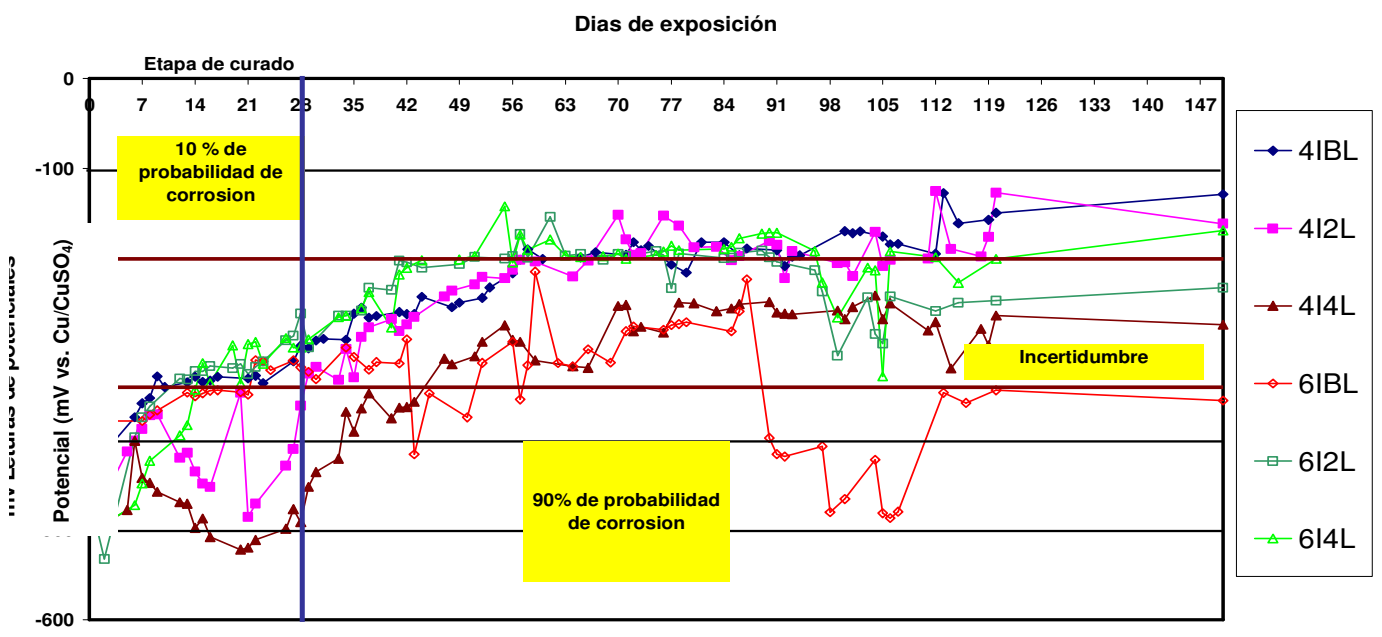

Figura 5. Especimenes expuestos en un medio limpio.

En la Fig. 6 se observa la influencia determinante del medio agresivo. Los valores encontrados presentan una tendencia en todos los especimenes, después de la etapa de curado a valores de potenciales más negativos conforme al paso del tiempo, a estar ubicados en un rango del $90 \%$ de probabilidad de corrosión de acuerdo a la norma ASTM C-876-91. Se puede observar también que los especimenes con relación 0.45 son los que presentan valores menos negativos $\mathrm{y}$, consecuentemente, son más resistentes al efecto del medio agresivo. 


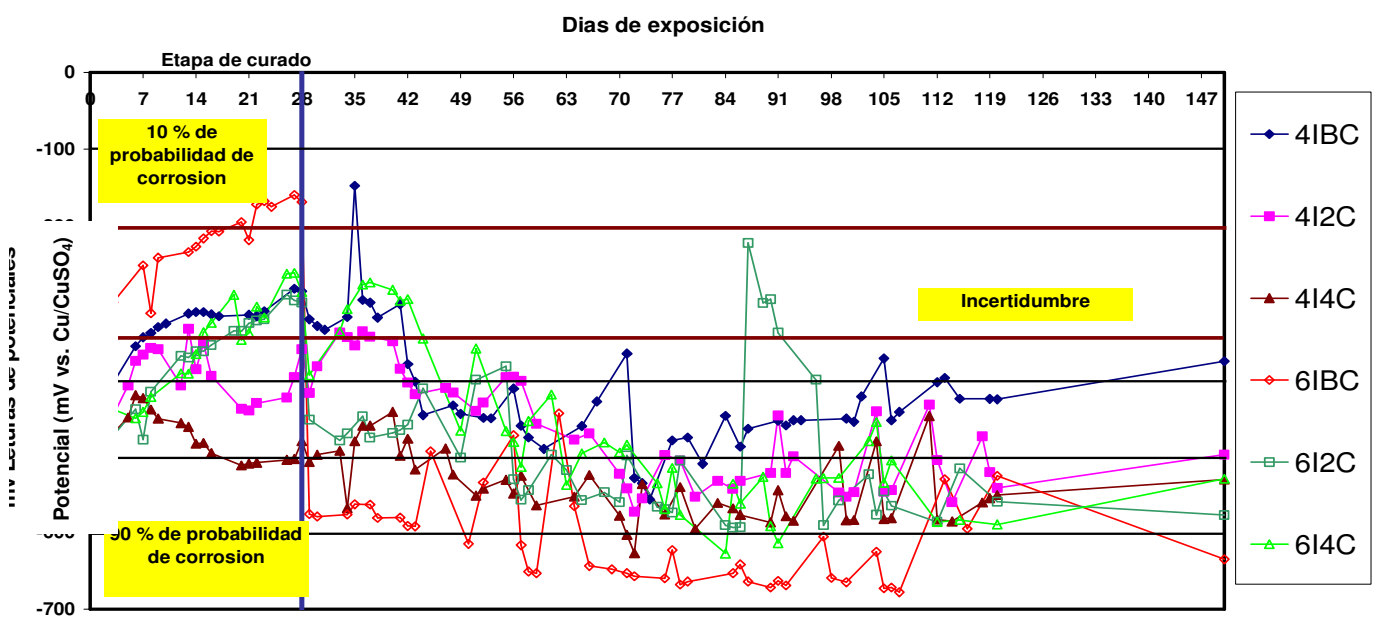

Figura 6. Especimenes expuestos en un medio al 3.5\% de $\mathrm{NaCl}$.

En la Fig. 7 se observa una tendencia de todos los especimenes a presentar valores de potenciales de corrosión más positivos conforme transcurre el periodo de exposición, con un comportamiento muy homogéneo de todos ellos. De acuerdo a la norma ASTM C-876-91 estos valores los sitúan con una probabilidad de corrosión del $10 \%$. Hasta este punto de la experimentación no se observa influencia alguna del tipo de cemento o del grado de contaminación del hormigón.

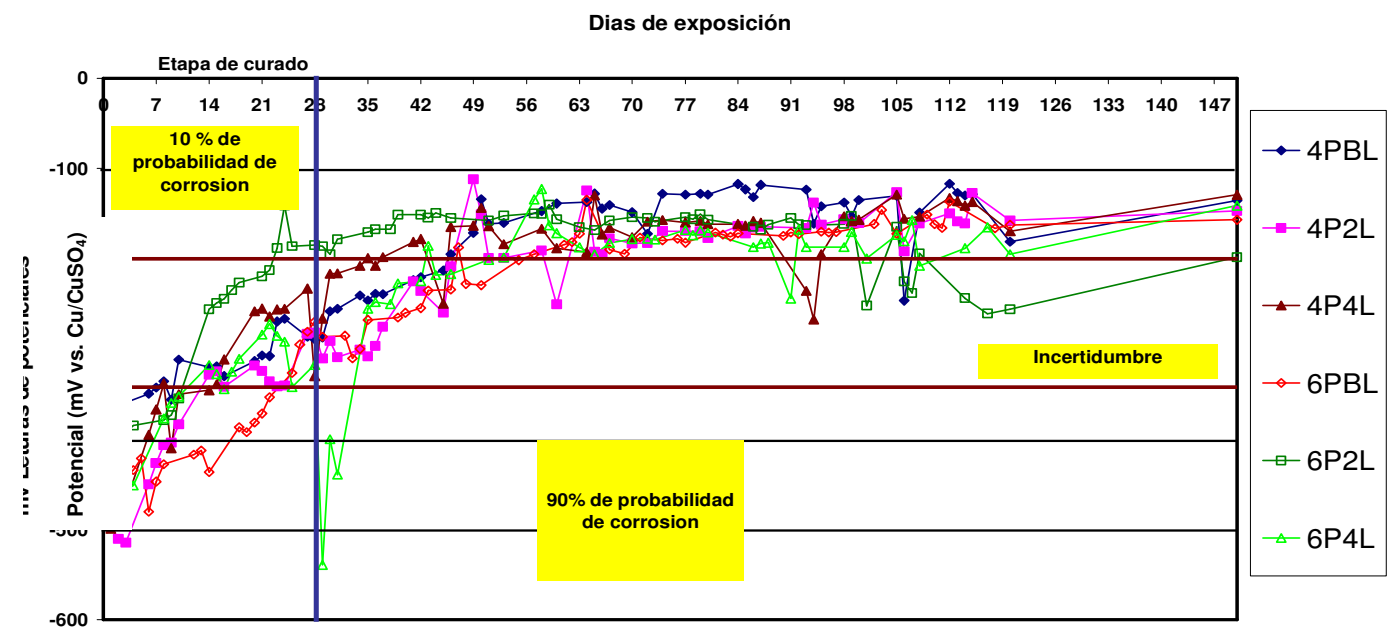

Figura 7. Especimenes expuestos en un medio limpio.

En la Fig. 8 también se observa, como en los especimenes de cemento impermeable, la influencia determinante del medio agresivo, presentando una tendencia en todos los especimenes, hacia valores que indican un $90 \%$ de probabilidad de corrosión. Si bien dos de ellos, en algún lapso de la experimentación, presentaron potenciales más nobles, al final del periodo ensayado caen en valores más negativos, lo que indica la presencia del fenómeno de corrosión. 


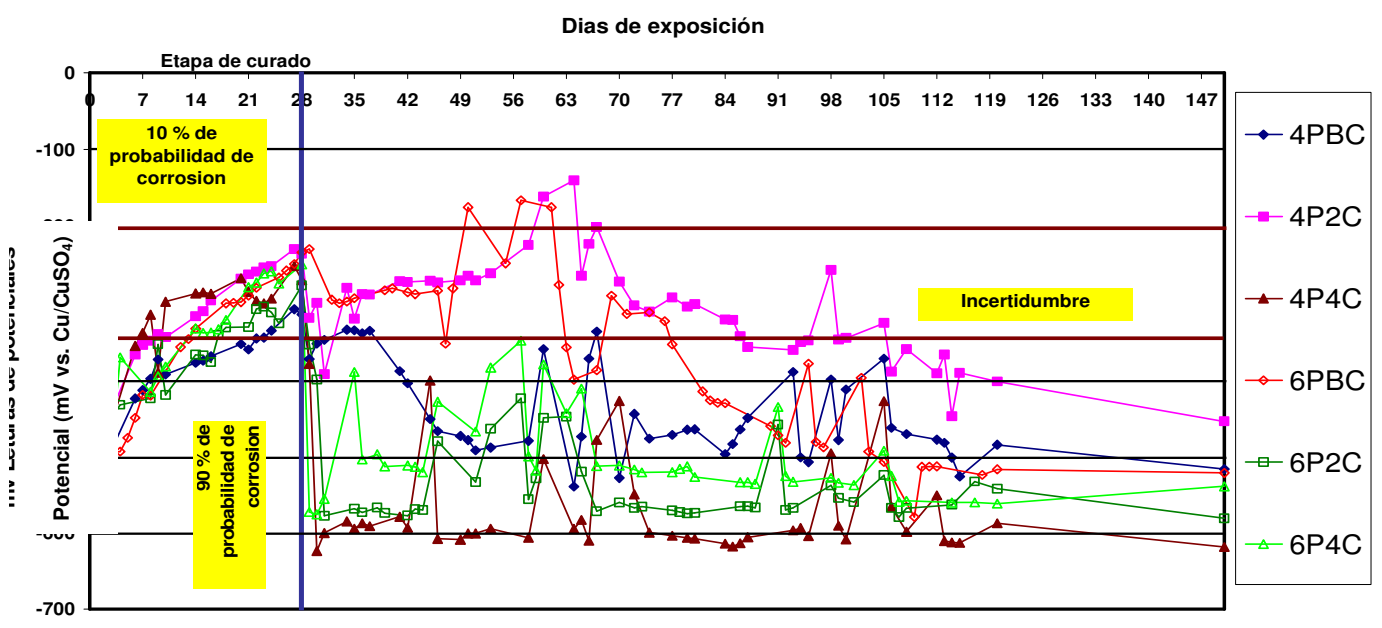

Figura 8. Especimenes expuestos en un medio al 3.5\% de $\mathrm{NaCl}$.

\section{Conclusiones}

\section{Potencial de corrosión}

- Los especimenes de cemento impermeable expuestos a un medio limpio presentan potenciales que indican incertidumbre de que exista corrosión, de acuerdo con la norma ASTM C-876-91. Se observa poca influencia del grado de contaminación de ambos hormigones. Los especimenes de cemento compuesto normal presentan un comportamiento mejor y más homogéneo que los de cemento impermeable, ubicándolos en un $10 \%$ de probabilidad de corrosión de acuerdo a la norma y observando una influencia casi nula de la relación a/c y del grado de contaminación del hormigón.

- Los especimenes de cemento impermeable expuestos a una disolución al 3.5\% de $\mathrm{NaCl}$ (medio agresivo) presentan, con el paso del tiempo, potenciales que indican un $90 \%$ de probabilidad de corrosión, de acuerdo con la norma ASTM C-876-91. Se observa poca influencia del grado de contaminación de ambos hormigones, teniendo los especimenes con relación a/c $=0.65$ los valores más negativos. Los especimenes de cemento compuesto normal también presentan, al transcurrir el tiempo de exposición, valores más negativos ubicando todos los especimenes con una probabilidad de corrosión del $90 \%$.

\section{Resistencia mecánica}

- La mayor resistencia a la compresión la presentaron los especimenes de relación a/c $=0.45$, teniendo mayor resistencia los especimenes elaborados con el cemento impermeable.

- La resistencia en los especimenes de relación a/c $=0.45$ contaminados con $2 \%$ de $\mathrm{NaCl}$, disminuye entre un $31 \%$ y un $44 \%$ y en los especimenes de $\mathrm{a} / \mathrm{c}=0.65$ entre un $25 \%$ y un $20 \%$.

- La resistencia en los especimenes de relación a/c $=0.45$ contaminados con $4 \%$ de $\mathrm{NaCl}$, disminuye de $30 \%$ á $49 \%$ y en los especimenes de relación a/c de 0.65 la resistencia disminuye de $20 \%$ á $45 \%$.

- El parámetro determinante en el presente estudio fue el medio agresivo, influyendo éste directamente en la probabilidad de corrosión del acero de refuerzo y disminuyendo la resistencia a la compresión de ambos hormigones. 


\section{Agradecimiento}

Se agradece a PROMEP el apoyo otorgado para la realización de este proyecto de investigación, autorizado por la SES mediante oficio no. PROMEP/103.5/07/2753, con cargo al "Proyecto de Apoyo a la Incorporación de Nuevos Profesores de Tiempo Completo PROMEP".

\section{Monitorización de potenciales de corrosión y resistencia mecánica de un hormigón contaminado expuesto a un medio clorado}

\section{Resumen}

Se evaluó el comportamiento electroquímico y mecánico de especimenes de hormigón limpio y contaminado con 2 y $4 \%$ de $\mathrm{NaCl}$ (de la cantidad de agua), elaborados con dos tipos de cemento: Compuesto e Impermeable. La dosificación de la mezcla de hormigón fue en base a peso con dos relaciones agua/cemento 0.45 y 0.65 . Los especimenes de estudio se expusieron en dos medios: agua limpia y disolución al 3\% de $\mathrm{NaCl}$. La prueba para evaluar la presencia de corrosión del acero de refuerzo, fue la monitorización de potenciales de media celda, como indica la norma ASTM C-876-91; para el comportamiento mecánico, se realizó la prueba a la compresión, según la norma ASTM C-39/C 39 - M - 99. El presente trabajo presenta los resultados de los primeros 4 meses de monitorización. No se observó una influencia importante del tipo de cemento en los parámetros de estudio, identificándose la influencia del ataque de los cloruros en la corrosión del acero y en sus propiedades mecánicas.

Palabras clave: hormigón, acero, corrosión, potenciales, cloruros.

\section{Referencias}

1. C.Q. Li, R.E. Melchers, W. Lawanwisut, Magazine of Concrete Research 57 (2005) 557.

2. Materials Performance (Supplement 4th July) (2002).

3. P. Gu, S. Elliot, J.J. Beaudoin, B. Arsenault, Corrosion Resistance of Stainless Steel in Chloride Contamined Concrete, Cement and Concrete Research 26 (8) (1996) 1151-1156.

4. G.S. Duffó, W. Morris, I. Raspini, C. Saragovi, A study of steel rebars embedded in concrete during 65 years, Corrosion Science 46 (2004) 21432157.

5. C. Andrade, Revista Iberoamericana de Corrosión y Protección 15 (4) (1984).

6. S.C. Kranc, A.A. Sagües, Polarization Current Distribution and Electrochemical Impedance Response of Reinforced Concrete when Using Guard Ring Electrodes, Electrochem. Acta 38 (14) (1993) 2055-2061.

7. C. Andrade, Matériaux et Constructions. RILEM 29, (1996) 40-46 and 97104. 
8. O.T. Rincón, Manual de Inspección, Evaluación y Diagnóstico de Corrosión en Estructuras de Hormigón Armado. Durar, $2^{\mathrm{a}}$ ed., Red Temática del CYTED, 1998.

9. C. Alonso, C. Andrade, M. Castellote, P. Castro, Chloride threshold values to depassivate reinforcing bars embedded in standardized OPC mortar, Cement and Concrete Research 30 (2000) 1047-1055.

10. P. Gu, S. Elliot, J.J. Beaudoin, B. Arsenault, Corrosion Resistance of Stainless Steel in Chloride Contamined Concrete, Cement and Concrete Research 26 (8) (1996) 1151-1156.

11. S. C. NMX-C-414-ONNCCE-2004, en Industria de la construcción Cementos hidráulicos - Especificaciones y métodos de prueba, Organismo Nacional de Normalización y Certificación de la Construcción y la Edificación, 2004.

12. Proporcionamiento de Mezclas, Concreto normal, pesado y masivo, ACI 211.1, IMCYC 2004, p. 29-34.

13. M.A. Baltazar, Corrosión por Sulfatos en Concreto Reforzado, Tesis de Maestría, Universidad Autónoma de Chihuahua, Chih., México, 2000.

14. M.A. Baltazar, F. Almeraya-Calderón, V. Orozco-Carmona, D. NievesMendoza, C. Gaona-Tiburcio, A. Borunda-Terrazas, A. Martínez-Villafañe, Evaluación de la Velocidad de Corrosión del Acero en Hormigón Expuesto a Soluciones de Sulfatos, Port. Electrochim. Acta 22 (4) (2004) 335-344.

15. Standard Test for Half-Cell Potentials of Uncoated Reinforcing steel in Concrete, in Annual Book of ASTM Standars, Philadelphia, 1992, Vol. 04.02, C876-91.

16. Standard Test Method for Compressive Strength of Cylindrical Concrete Specimens, in Annual Book of ASTM Standars, Philadelphia, 1992, Vol. 4.02, C39/C39M-04.

17. Z.M. Baltazar, Comportamiento de la corrosión del acero al carbono e inoxidable embebido en concreto y en presencia de $\mathrm{NaCl}$ y $\mathrm{Na}_{2} \mathrm{SO}_{4}$, Tesis de Doctorado, Centro de Investigación en Materiales Avanzados, S.C. Chihuahua, Chih., México, 2005. 